\title{
Inclusión de musgo (Sphagnum magellanicum Brid.) y fibra de coco como componentes orgánicos del sustrato para almácigos de kiwi (Actinidia deliciosa)
}

\author{
Inclusion of moss (Sphagnum magellanicum Brid.) and coconut \\ fiber as organic components for kiwi (Actinidia deliciosa) \\ María Eugenia Arévalo P. ${ }^{1 *}$, Christel Oberpaur $W^{2}{ }^{2}$ Cristian Méndez $C .^{2}$
}

\begin{abstract}
RESUMEN
Diversos materiales pueden ser utilizados como sustratos, sin embargo, es común que no se escoge el más apropiado, pudiendo además generarse un deterioro ambiental por su extracción como es el caso de la turba. En la búsqueda de alternativas ecosustentables se evaluó la incorporación de musgo y fibra de coco como componentes orgánicos del sustrato para mejorar la producción de almácigo de kiwi, propagado por semilla en contenedor. Se consideró la inclusión del musgo Sphagnum magellanicum (SM), fibra de coco (FC), corteza de pino compostada (CP) y arena (A), además de turba (T) en diferentes proporciones. El estudio se realizó bajo condiciones de invernadero, en Paine, Región Metropolitana, Chile. Se evaluaron 16 diferentes mezclas de sustratos en proporciones en base a volumen: $\mathrm{T} 40 \%+\mathrm{CP} 40 \%+\mathrm{A} 20 \%$ (control), $\mathrm{SM} 40 \%+\mathrm{FC} 40 \%+\mathrm{A} 20 \%, \mathrm{SM} 40 \%+\mathrm{T} 40 \%+\mathrm{A} 20 \%$, $\mathrm{SM} 40 \%+\mathrm{CP} 40 \%+\mathrm{A} 20 \%, \mathrm{FC} 40 \%+\mathrm{T} 40 \%+\mathrm{A} 20 \%, \mathrm{FC} 40 \%+\mathrm{CP} 40 \%+\mathrm{A} 20 \%, \mathrm{SM} 80 \%+\mathrm{A} 20 \%, \mathrm{FC} 80 \%+\mathrm{A} 20 \%, \mathrm{~T} 80 \%+$ $\mathrm{A} 20 \%, \mathrm{CP} 80 \%+\mathrm{A} 20 \%, \mathrm{SM} 33 \%+\mathrm{FC} 33 \%+\mathrm{A} 33 \%, \mathrm{SM} 33 \%+\mathrm{T} 33 \%+\mathrm{A} 33 \%, \mathrm{SM} 33 \%+\mathrm{CP} 33 \%+\mathrm{A} 33 \%, \mathrm{FC} 33 \%+\mathrm{T} 33 \%+$ $\mathrm{A} 33 \%, \mathrm{FC} 33 \%+\mathrm{CP} 33 \%+\mathrm{A} 33 \%, \mathrm{~T} 33 \%+\mathrm{CP} 33 \%+\mathrm{A} 33 \%$. En todos los sustratos se sembró semillas de kiwi cv. Hayward. El diseño estadístico fue en bloques completos al azar, con cuatro repeticiones. Las variables evaluadas fueron diámetro del tallo, peso seco radicular y aéreo por planta. La unidad experimental del ensayo consideró 22 tubetes de $120 \mathrm{~cm}^{3}$, con una planta en cada uno. Los resultados indican que los sustratos compuestos por $80 \%$ de musgo Sphagnum mezclado con un $20 \%$ de arena y aquel con un $80 \%$ de fibra de coco combinado con un $20 \%$ de arena, en comparación al sustrato control, son adecuados para la producción de almácigos de kiwi.
\end{abstract}

Palabras clave: turba, corteza de pino, fibra de coco.

\begin{abstract}
Several components are commonly included in substrate mixtures, but usually they are not the most appropriated; some of them, due to their extraction, generate a negative environmental damage, as it's the case of peat. Searching for sustainable alternatives, the inclusion of Sphagnum magellanicum moss and coconut fiber as organic components of substratum mixtures was evaluated in order to improve the nursery production of kiwi, propagated by seeds, in plugs. The considered components were Sphagnum moss $(S M)$, coconut fiber $(F C)$, composted pine bark $((C P)$ and sand $(A)$, further peat $(T)$ in different proportions. The trial was realized in Paine, Metropolitan Region, Chile, under greenhouse conditions. Sixteen mixtures were evaluated: T40\% $+C P 40 \%+A 20 \%$ (control), SM40\% + FC40\% +A20\%,SM40\% + T40\% +A20\%, SM40\%+CP40\%+A20\%, FC40\%+T40\%+A20\%, FC40\% $+C P 40 \%+A 20 \%, S M 80 \%+A 20 \%, F C 80 \%+A 20 \%, T 80 \%+A 20 \%, C P 80 \%+A 20 \%, S M 33 \%+F C 33 \%+A 33 \%, S M 33 \%$ $+T 33 \%+A 33 \%, S M 33 \%+C P 33 \%+A 33 \%, F C 33 \%+T 33 \%+A 33 \%, F C 33 \%+C P 33 \%+A 33 \%, T 33 \%+C P 33 \%+A 33 \%$. In all the substrata, kiwi seeds $c v$. Hayward were sawn in containerized plugs. The statistical design corresponded to complete randomized blocks, with four repetitions. Evaluated variables were: stem diameter, root and canopy dry weight. The experimental unit considered 22 plug container tubes of $120 \mathrm{~cm}^{3}$, each one with a single plant. The results indicate that the substrata composed by $80 \%$ of Sphagnum moss and $20 \%$ of sand, or $80 \%$ of coconut fiber and $20 \%$ of sand in comparison to the control substratum is appropriate for the production of kiwi seedlings and constitute an alternative as replacement to the use of peat.
\end{abstract}

Key words: peat, composted pine bark, coconut fiber.

\footnotetext{
1 Asociación Gremial de Viveros de Chile. Napoleón 3565, oficina 202, Las Condes, Chile.

2 Escuela de Agronomía, Universidad Santo Tomás, Ejército 146, Santiago, Chile.

* Corresponding author: marevalo@ viverosfrutales.cl
}

Fecha de Recepción: 19 Enero, 2015.

Fecha de Aceptación: 23 Febrero, 2016. 


\section{Introducción}

Un sustrato es todo material sólido distinto del suelo, natural o sintético, mineral u orgánico, que colocado en un contenedor, en forma pura o en mezcla, permite el anclaje del sistema radical desempeñando, por lo tanto, un papel de soporte para la planta (Honorato y Bonomelli, 1999). Un sustrato es el medio material donde se desarrolla el sistema radicular del cultivo, donde sus funciones básicas son proveer agua y nutrientes, permitir el intercambio gaseoso, desde y hacia la raíz, y brindar soporte a la planta. No obstante, se ha observado que las propiedades físicas cobran mayor relevancia respecto de las demás, debido a que estas una vez colocado el material vegetal en la maceta son difíciles de modificar (Pastor, citado por CruzCrespo et al., 2012).

El cultivo de plantas en sustrato difiere marcadamente del cultivo en suelo, porque al usar contenedores el volumen del medio de cultivo es limitado, puesto que las raíces no pueden extenderse fuera de él para absorber el agua, oxígeno y elementos nutritivos. Con estas restricciones, el mejor sustrato será aquel que proporcione la máxima cantidad de agua, el mayor volumen de aire, los elementos nutritivos necesarios, el anclaje adecuado para las raíces y que además no contenga ningún componente que frene el crecimiento de la planta (Bures, 2002). Los componentes básicos de los sustratos son generalmente turba, perlita, vermiculita y un agente humectante, otros ingredientes pueden ser arenas lavadas, corteza procesada, residuos vegetales sometidos a compostaje u otros biosólidos. La mayoría de estos son harneados para facilitar el llenado de celdas pequeñas, así como para obtener una adecuada germinación de las semillas (Schrader, 2003). Para la elaboración de sustratos, la elección de los componentes dependerá de la especie vegetal a propagar, tipo de propágulo, época, sistema de propagación, precio, disponibilidad y características propias del sustrato (Hartmann et al., 2002). La elección debe considerar el tamaño y forma del contenedor, sistema de riego y fertilización, aspectos económicos, experiencia local en su utilización, entre otros (Abad, 1995).

De acuerdo a Baudoin et al. (2002) y Ansorena (1994) un sustrato ideal, debería mostrar una densidad aparente de $220 \mathrm{~kg} \mathrm{~m}^{-3}$, un $\mathrm{pH}$ entre 5,5 y 6,5 , un nivel de CIC de 10 a 30 meq $100 \mathrm{~g}^{-1}$, conductividad eléctrica de 0,75 a $3,49 \mathrm{dS} \mathrm{m}^{-1}$ y más de $80 \%$ de materia orgánica. El valor óptimo de porosidad para un sustrato es superior al $85 \%$. Existen importantes diferencias en la capacidad de intercambio gaseoso al modificar la naturaleza de los materiales del sustrato, el valor óptimo se sitúa entre 20 y $30 \%$ (Baixauli y Aguilar, 2002).

Las principales propiedades físicas para caracterizar un sustrato son densidad aparente, densidad real, granulometría, porosidad total, de aire y contenido de agua, que se compone de: agua fácilmente disponible, de reserva y agua difícilmente disponible (Díaz, 2004). Pastor (2003), indica que la capacidad de retención de agua fácilmente disponible, deberá ser de un 20 a un $30 \%$, como valor óptimo.

Es deseable que un sustrato sea químicamente estable, con baja o nula salinidad, de $\mathrm{pH}$ neutro o ligeramente ácido y con una adecuada relación $\mathrm{C} / \mathrm{N}$ (Baixauli y Aguilar, 2002). El valor adecuado para la CIC de los sustratos depende estrechamente de la frecuencia de la fertirrigación (Lemaire et al., citados por Abad, 1995), por lo que si se aplica permanentemente es recomendable la utilización de materiales inertes con muy baja o nula CIC. Si, por el contrario, se aplica de forma intermitente es interesante la utilización de sustratos con moderada o elevada CIC, superior a 20 meq $100 \mathrm{~g}^{-1}$ (Abad, 1993). Hartmann y Kester et al. (2002) establecen que el exceso de sales en el sustrato de propagación se considera cuando supera $2 \mathrm{dS} \mathrm{m}^{-1}$ y puede reducir el crecimiento de las plantas, producir quemaduras de hojas y, eventualmente, causar la muerte de estas.

La turba mejora la retención hídrica en un sustrato por su gran capacidad de absorción de agua, manteniéndolo con un alto contenido de humedad y alta porosidad, mejora la aireación. Forma una estructura tipo granular, crea una alta CIC (Hartmann et al., 2002), que oscila entre 130 y 200 meq $100 \mathrm{~g}^{-1}$ de turba seca, variando desde turbas rubias a negras respectivamente (Jeangille, 1998).

La corteza de pino es un sustrato liviano, generalmente libre de patógenos, de buen drenaje. Constituye un buen sustituto de la turbas, por lo que es utilizada como componente orgánico en los sustratos para contenedores de numerosas plantas leñosas y ornamentales (Hartmann et al., 2002). Como componente del medio es incluida generalmente en cantidades de hasta $50 \%$ del volumen (Schmilewski, 2008).

El musgo Sphagnum magellanicum posee una gran capacidad de absorción de agua, hasta 20 
veces su peso seco (Schofield, 1985). Gaudig et al. (2014), indican que la biomasa fresca de Sphagnum constituye la alternativa más prometedora al uso de turba en los sustratos, pues presenta propiedades físicas y químicas similares a la turba rubia.

La fibra de coco es un desecho de la industria alimentaria de procesado de coco. Entre sus características se cuentan las siguientes: elevada porosidad $(64,1-98,3 \%)$, baja densidad aparente $\left(0,03-0,9 \mathrm{~g} \mathrm{~cm}^{-3}\right)$, aireación $(24,2-89,4 \%)$, capacidad de retención de agua (137-786 $\mathrm{mL} \mathrm{L}^{-1}$ de sustrato), donde estos valores dependen del tamaño de las partículas (Abad et al., 2005). Su CIC varía entre 38,6 y $7,6 \mathrm{cmol} \mathrm{kg}^{-1}$, el $\mathrm{pH}$ entre 4,9 y 6,1 ; y la conductividad eléctrica entre 0,4 y $4,5 \mathrm{dS} \mathrm{m}^{-1}$, donde iunfluye marcadamente su procedencia (Abad et al., 2002). La arena es un material de alta densidad $\left(1,5 \mathrm{~g} \mathrm{~cm}^{-3}\right)$, proveniente de ríos o extraída de canteras, cuyo tamaño de partículas es de 0,5-2 mm. Generalmente se utiliza para mejorar drenaje de otros sustratos y para aumentar la estabilidad del contenido. Posee una aireación aceptable con un $40 \%$ de porosidad total (Jeangille, 1998).

El kiwi se adapta a variados tipos de suelos, prefiriendo los de textura mediana a liviana (Sudzuki, 2002). Exige suelos ligeros, frescos y ricos en humus, es muy sensible a excesos de humedad, por lo que el agua debe drenar rápidamente (Zuccherelli y Zuccherelli, 1990; Sudzuki, 2002). En general se adapta muy mal a suelos arcillosos y pesados. En granulometría requiere al menos un $11 \%$ de partículas gruesas, un 53\% de arena fina, de 10 a $15 \%$ de limo y $10 \%$ de arcilla. Requiere un $\mathrm{pH}$ neutro a subácido, de 6 a 7,0, tolerando hasta 7,5; sin embargo, presenta síntomas de clorosis férrica con $\mathrm{pH} 8$ y la caliza activa no debe superar el $5 \%$. El aporte de materia orgánica es muy favorable y recomendable para esta especie, manifestándose en un notable y rápido aumento del vigor (Zuccherelli y Zuccherelli, 1990). La obtención de los portainjertos de esta especie se realiza a través de semillas, e injertando posteriormente sobre estos el cultivar comercial, de esta forma se obtiene un mejor sistema radicular (Sudzuki, 2002).

La investigación de nuevos materiales para formular sustratos cada vez más específicos que sirvan como medio de crecimiento vegetal es fundamental, debido al encarecimiento y baja disponibilidad de los ya existentes. Por ello es relevante investigar la aptitud de los subproductos o residuos generados por diferentes actividades productivas y de consumo como componentes de sustratos, particularmente por su reducido costo (Zapata et al., 2005). Los objetivos de esta investigación son determinar cómo afecta la incorporación de musgo Sphagnum magellanicum y fibra de coco como componentes orgánicos del sustrato para la producción de almácigo de kiwi en contenedor; y establecer las principales propiedades físicas y químicas de los sustratos en condiciones de laboratorio para compararlas con el usado actualmente en viveros.

\section{Materiales y Métodos}

La preparación de mezclas de sustratos con incorporación de musgo, se realizó en los Laboratorios de Ciencias Naturales y Bioquímica de la Universidad Santo Tomás, comuna de Santiago, Región Metropolitana. La siembra y manejo de kiwis en contenedores se efectuó bajo condiciones de invernadero frío, tipo túnel. El ensayo se realizó en las dependencias de Univiveros (Sociedad Agrícola Uniagri Copiapó Ltda.), comuna de Paine, Región Metropolitana. El experimento se prolongó desde el 25 de octubre de 2008 hasta el 30 de marzo de 2009.

Los materiales utilizados para las respectivas mezclas de sustratos fueron desechos de exportación de Sphagnum magellanicum, fibra de coco (Growshop), turba (Kekkila), corteza de pino compostada (Gromor) y arena y Basacote Plus ${ }^{\circledR}$ $3 \mathrm{M}$. Este producto comercial corresponde a gránulos de 2 a $4 \mathrm{~mm}$ recubiertos de ceras elásticas para la liberación controlada de los nutrientes (Compo Agro, 2014).

Los tratamientos, que corresponden a los distintos sustratos (Tabla 1) son elaborados en base a volumen y en todas las mezclas se incorporó el fertilizante Basacote Plus $3 \mathrm{M}$ a una dosis de $3 \mathrm{~kg}$ $\mathrm{m}^{-3}$ de sustrato.

Equipos. pH-metro Gondo. Modelo PP-203K \# serie 945475, Conductivímetro HI9033 (rango 0,00-19,99 dS m ${ }^{-1}$; Precisión +/- 0,19 dS m ${ }^{-1}$ ); Digital lab scale. LTECH 300 (rango 0,0000-300 g; Precisión +/- 0,01 g), estufa de secado Binder (rango 0-300 ${ }^{\circ} \mathrm{C}$ ); horno mufla Vulcan A-550 (rango 0-1100 ${ }^{\circ} \mathrm{C}$ ); agitador eléctrico. VRN (0-250 rpm); agitador eléctrico Magnetic stiner, Modelo ST5; desecador; harneros de $7 \mathrm{~mm}$; pie de metro manual.

Preparación de los sustratos. El musgo Sphagnum seco se tamizó con un harnero de $7 \mathrm{~mm}$ 
Tabla 1. Mezclas de sustratos para la siembra de portainjerto de kiwi.

\begin{tabular}{|c|c|c|c|c|c|}
\hline $\begin{array}{l}\text { Tratamiento } \\
\text { (Sustrato) }\end{array}$ & $\begin{array}{c}\text { Sphagnum (SM) } \\
(\%)\end{array}$ & $\begin{array}{l}\text { Fibra de coco }(\mathrm{FC}) \\
\qquad(\%)\end{array}$ & $\begin{array}{l}\text { Turba (T) } \\
(\%)\end{array}$ & $\begin{array}{c}\text { Corteza de pino }(\mathrm{CP}) \\
(\%)\end{array}$ & $\begin{array}{c}\text { Arena }(\mathrm{A}) \\
(\%)\end{array}$ \\
\hline T40CP40A20* & & & 40 & 40 & 20 \\
\hline SM40FC40A20 & 40 & 40 & & & 20 \\
\hline SM40T40A20 & 40 & & 40 & & 20 \\
\hline SM40CP40A20 & 40 & & & 40 & 20 \\
\hline FC40T40A20 & & 40 & 40 & & 20 \\
\hline FC40CP40A20 & & 40 & & 40 & 20 \\
\hline SM80A20 & 80 & & & & 20 \\
\hline FC80A20 & & 80 & & & 20 \\
\hline T80A20 & & & 80 & & 20 \\
\hline CP80A20 & & & & 80 & 20 \\
\hline SM33FC33A33 & 33 & 33 & & & 33 \\
\hline SM33T33A33 & 33 & & 33 & & 33 \\
\hline SM33CP33A33 & 33 & & & 33 & 33 \\
\hline FC33T33A33 & & 33 & 33 & & 33 \\
\hline FC33CP33A33 & & 33 & & 33 & 33 \\
\hline T33СР33A33 & & & 33 & 33 & 33 \\
\hline
\end{tabular}

* Control. Corresponde a la mezcla que se utiliza en el vivero al momento del experimento.

para eliminar impurezas, luego se regó una vez con agua recién hervida para prevenir la presencia de posibles malezas. El musgo fue utilizado una vez enfriado y seco. Posteriormente se elaboraron los sustratos compuestos por musgo Sphagnum, fibra de coco, corteza de pino, turba y arena, agregando a la respectiva mezcla el fertilizante de entrega controlada.

Manejo del experimento. En el ensayo se utilizaron 16 bandejas plásticas con capacidad para 88 tubetes, cada uno con un volumen de 120 $\mathrm{cm}^{3}$. Una vez formuladas las mezclas se sembró en los contenedores. Para la obtención de semillas se maceró la pulpa de los frutos de kiwi de calibre 36, maduros, se eliminó mediante triple lavado restos de ella. Las semillas se desinfectaron remojando por un minuto en una dilución de hipoclorito de sodio al $2 \%$ y Captan $80 \mathrm{WP}$ al $0,15 \%$, agitando en forma permanente. Las semillas escurridas se pesaron en dosis fijas por bandeja de estratificación, envueltas en una doble capa de papel absorbente y posteriormente se cubrió con plástico y se almacenó a una temperatura 2 a $4{ }^{\circ} \mathrm{C}$ durante 15 días. Antes de la siembra se desinfectó nuevamente la semilla por inmersión de 10 minutos en la solución desinfectante. Se sembraron 6 semillas por tubete, cubriéndolas con una capa de $2 \mathrm{~cm}$ del sustrato correspondiente. A los 14 días después de la emergencia se ralearon las plantas en el estado de primer par de hojas verdaderas, dejando una sola por tubete, El riego del ensayo fue mediante aspersión manual con una frecuencia diaria, sin fertirrigación.

De acuerdo a resultados del análisis de varianza, al finalizar el ensayo, se envió a un laboratorio comercial de análisis oficial muestras de los cinco mejores sustratos originales, sin fertilizante, para su análisis fisicoquímico. A su vez al finalizar el ensayo se midió $\mathrm{pH}$ y conductividad eléctrica en todos los sustratos utilizados, que incluían el fertilizante de entrega controlada.

\section{Evaluaciones}

Las evaluaciones se realizaron al finalizar el ensayo, seleccionando 10 plantas al azar por unidad experimental. Las variables evaluadas fueron diámetro del tallo, determinado a la mitad de la distancia entre el primer par de hojas y la inserción de las raíces, peso seco de la planta (aéreo y radicular) luego de secar a estufa, $105^{\circ} \mathrm{C}$ durante 24 horas.

\section{Diseño experimental y análisis estadístico}

El diseño experimental correspondió a bloques completos al azar, con 16 tratamientos y 4 repeticiones. La unidad experimental del ensayo consideró 22 tubetes cada uno con una planta. Los resultados se evaluaron mediante análisis de varianza utilizando el software estadístico MINITAB Release 13.31, 2000. Los tratamientos que presentaron diferencias estadísticamente significativas se 
sometieron a la prueba de comparación múltiple de Tukey $(\mathrm{p} \leq 0,05)$.

\section{Resultados y Discusión}

\section{Diámetro de plántulas}

En la Tabla 2 los resultados obtenidos para esta variable en plántulas de kiwi señalan que solo existen diferencias significativas entre los tratamientos FC40T40A20 y FC80A20, donde el primero el más favorable, probablemente a consecuencia de la inclusión de turba en este sustrato. Entre los demás tratamientos no se determinaron diferencias significativas. Olivo y Buduba (2006) describen un buen resultado en la producción de plantas forestales, especialmente cuando la fibra de coco es mezclada con sustratos inorgánicos, situación en que las plantas presentan características morfológicas de diámetro de cuello, altura total y biomasa adecuadas. Según Roselló et al. (1999) la fibra de coco presenta problemas debido a su alta acidez, pero puede ser un sustituto aceptable de la turba ya que demuestra menor compactación y pérdida de volumen. La turba dada su alta porosidad, mejora la aireación, impide la falta de oxígeno y exceso de dióxido de carbono, facilitando el crecimiento de las plantas (Hartmann et al., 2002).

\section{Peso seco radical, aéreo y total}

De acuerdo con la Tabla 2, el peso seco radical en las plántulas mostró diferencias significativas entre los sustratos SM40CP40A20 y FC40CP40A20, respecto a SM80A20, correspondiendo a este último el mayor valor de peso seco radical. Los sustratos SM40CP40A20 y FC40CP40A20 presentaron los menores valores, por lo que probablemente la inclusión de corteza de pino compostada en una proporción de $40 \%$ afecta negativamente el peso radical; sin embargo, no se diferencia significativamente del control.

La alternativa más prometedora para reemplazar la turba en medios de cultivo es el musgo Sphagnum, debido a que presenta características físicas y químicas similares a la turba rubia (Gaudig et al., 2014). La turba se caracteriza por poseer una elevada capacidad de retención de agua, mantiene la humedad en el sustrato, mejora además su densidad aparente y porosidad (Li et al., 2004). En este ensayo el mejor sustrato se obtuvo al combinar el musgo con arena, complementado con una fertilización base inicial. La inclusión de un $40 \%$ de corteza compostada en las mezclas junto con fibra de coco o musgo Sphagnum, acompañado por un $20 \%$ de arena, resultan en menores pesos radicales, sin diferenciarse de las demás mezclas (con excepción

Tabla 2. Influencia de las mezclas de sustrato sobre plántulas de kiwi en vivero.

\begin{tabular}{lcccc}
\hline Mezclas & $\begin{array}{c}\text { Diámetro } \\
\left(\mathrm{mm} \mathrm{planta}^{-1}\right)\end{array}$ & $\begin{array}{c}\text { Peso seco radical } \\
\left(\mathrm{g} \mathrm{planta}^{-1}\right)\end{array}$ & $\begin{array}{c}\text { Peso canopia } \\
\left(\mathrm{g} \mathrm{planta}^{-1}\right)\end{array}$ & $\begin{array}{c}\text { Peso seco total } \\
\left(\mathrm{g} \mathrm{planta}^{-1}\right)\end{array}$ \\
\hline FC40T40A20 & $4,05 \mathrm{a}$ & $0,84 \mathrm{ab}$ & $0,54 \mathrm{ab}$ & $1,38 \mathrm{ab}$ \\
SM40T40A20 & $3,98 \mathrm{ab}$ & $0,98 \mathrm{ab}$ & $0,61 \mathrm{ab}$ & $1,59 \mathrm{ab}$ \\
SM80A20 & $3,90 \mathrm{ab}$ & $1,31 \mathrm{a}$ & $0,65 \mathrm{ab}$ & $1,96 \mathrm{a}$ \\
T80A20 & $3,89 \mathrm{ab}$ & $0,80 \mathrm{ab}$ & $0,72 \mathrm{a}$ & $1,52 \mathrm{ab}$ \\
FC33T33A33 & $3,86 \mathrm{ab}$ & $0,94 \mathrm{ab}$ & $0,49 \mathrm{ab}$ & $1,16 \mathrm{ab}$ \\
SM33T33A33 & $3,82 \mathrm{ab}$ & $0,63 \mathrm{ab}$ & $0,53 \mathrm{ab}$ & $1,09 \mathrm{ab}$ \\
T40CP40A20 & $3,81 \mathrm{ab}$ & $0,57 \mathrm{ab}$ & $0,52 \mathrm{ab}$ & $1,03 \mathrm{ab}$ \\
SM33FC33A33 & $3,59 \mathrm{ab}$ & $0,58 \mathrm{ab}$ & $0,45 \mathrm{ab}$ & $1,02 \mathrm{ab}$ \\
SM33CP33A33 & $3,52 \mathrm{ab}$ & $0,54 \mathrm{ab}$ & $0,48 \mathrm{ab}$ & $0,91 \mathrm{ab}$ \\
T33CP33A33 & $3,49 \mathrm{ab}$ & $0,55 \mathrm{ab}$ & $0,36 \mathrm{ab}$ & $0,84 \mathrm{ab}$ \\
FC33CP33A33 & $3,47 \mathrm{ab}$ & $0,54 \mathrm{ab}$ & $0,30 \mathrm{ab}$ & $1,20 \mathrm{ab}$ \\
SM40FC40A20 & $3,42 \mathrm{ab}$ & $0,77 \mathrm{ab}$ & $0,42 \mathrm{ab}$ & $0,65 \mathrm{~b}$ \\
SM40CP40A20 & $3,41 \mathrm{ab}$ & $0,29 \mathrm{~b}$ & $0,36 \mathrm{ab}$ & $0,62 \mathrm{~b}$ \\
FC40CP40A20 & $3,30 \mathrm{ab}$ & $0,35 \mathrm{~b}$ & $0,27 \mathrm{~b}$ & $0,94 \mathrm{ab}$ \\
CP80A20 & $3,10 \mathrm{ab}$ & $0,49 \mathrm{ab}$ & $0,45 \mathrm{ab}$ & $0,82 \mathrm{ab}$ \\
FC80A20 & $3,05 \mathrm{~b}$ & $0,50 \mathrm{ab}$ & $0,32 \mathrm{ab}$ & \\
\hline
\end{tabular}

Promedios con distinta letra en la columna, indican diferencias significativas entre tratamientos, según prueba de Tukey $(\mathrm{p} \leq 0,05)$. T (turba); CP (corteza de pino); SM (Sphagnum); FC (fibra de coco); A (arena).

*Control. Corresponde a la mezcla que se utiliza en el vivero al momento del experimento. 
de SM80A20). De acuerdo con estudios de la década de los 90 de Arrieta y Terés (1993), quienes caracterizaron la corteza de pino y sus propiedades físicas y químicas, describen bajos niveles de $\mathrm{N}$ $(0,65 \%$ de $\mathrm{N}$ total $)$ y $\mathrm{P}\left(0,004 \%\right.$ de $\left.\mathrm{P}_{2} \mathrm{O}_{5}\right)$. Esto amerita la fertilización de base con este elemento, que en este caso fue de entrega controlada. A pesar de esto, las mezclas que contenían corteza de pino obtuvieron los menores pesos secos radicales, lo que se debería a la deficiencia nutricional de la corteza de pino respecto a los otros componentes orgánicos de las mezclas.

Los resultados de peso seco aéreo (Tabla 2), evidencian diferencias significativas entre los sustratos FC40CP40A20 y SMCP40A20 respecto a T80A20, donde en este último se obtuvo el mayor valor de peso seco aéreo y en C40CP40A20 el menor valor, lo que es coincidente con lo logrado en peso seco radical. Al igual que en peso seco radical, el sustrato con un $80 \%$ de turba favorece el peso seco aéreo. Hartmann y Kester et al. (2002) señalan a la turba como el mejor sustrato orgánico y el más utilizado, por la estructura de tipo granular que presenta, por sus características de alta porosidad y buena aireación, favoreciendo el crecimiento de raíces y, por ende, el desarrollo de la parte aérea de la planta. La turba también contiene ácidos húmicos que se forman como resultado de la descomposición de la lignina y otras sustancias similares que influyen positivamente en las plantas (Puustjärvi, 1994). El efecto de los sustratos ensayados sobre el peso seco total de plántulas de kiwi (Tabla 2), indica diferencias significativas entre los sustratos SM40CP40A20 y FC40CP40A20 respecto a SM80A20, donde el peso seco total fue mayor. El sustrato testigo no se diferencia de las demás mezclas. La inclusión de corteza de pino compostada afectaría negativamente a las plantas independientemente de los componentes con que se haya combinado, por el contrario la turba mostró mejores resultados en combinación con los demás sustratos. Al igual que en peso seco aéreo y peso seco radical, la inclusión del musgo Sphagnum obtuvo rendimientos adecuados en combinación con arena, o en combinación con turba, lo que sugiere que constituye una alternativa de remplazo a la turba en un vivero de kiwi.

El sustrato testigo se ubica en la medianía de la Tabla 2, tanto en peso seco total, peso seco aéreo y radical como en el diámetro, esto indica que en ese sustrato las plántulas no logran su potencial. La presencia de toxinas y sustancias inhibidoras de crecimiento presentes en la corteza de pino corresponden a un factor a considerar en su uso. Posiblemente, debido a la falta de nutrientes de base y a posibles restos de inhibidores en la corteza de pino compostada, en los sustratos SM40CP40A20 y FC40CP40A20 se obtuvieron los menores valores de peso seco total.

\section{Análisis fisicoquímico de los sustratos}

Una vez realizado el análisis estadístico de los resultados se seleccionó las siguientes sustratos además del control: SM80A20, FC80A20, T80A20, CP80A20 y T40CP40A20 (Control), sin incluir fertilizante de entrega controlada. Los valores de $\mathrm{pH}$ en la Tabla 3 indican que el sustrato FC80A20 es el único que se encuentra en el nivel óptimo indicado por Baudoin et al. (2002), en cambio las mezclas T40CP40A20, SM80A20 y CP80A20 están por debajo del rango, con valores muy ácidos. En el caso de la mezcla T80A20 el valor es más alcalino que el óptimo. Según Ansorena (1994) a un pH muy

Tabla 3. Resultados de los análisis físicos y químicos de algunos sustratos promisorios para un vivero de kiwi.

\begin{tabular}{|c|c|c|c|c|c|c|c|}
\hline Sustratos & $\mathrm{pH}$ & 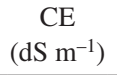 & $\begin{array}{l}\text { M.O. } \\
(\%)\end{array}$ & $\begin{array}{l}\mathrm{N} \\
(\%)\end{array}$ & $\begin{array}{l}\mathrm{P}_{2} \mathrm{O}_{5} \\
(\%)\end{array}$ & $\begin{array}{l}\mathrm{K}_{2} \mathrm{O} \\
(\%)\end{array}$ & $\begin{array}{c}\mathrm{Da} \\
\left(\mathrm{kg} \mathrm{m}^{-3}\right)\end{array}$ \\
\hline T40CP40A20 (control) & 5,3 & 0,6 & 20,0 & 0,29 & 0,25 & 0,06 & 460 \\
\hline SM80A20 & 5,0 & 0,4 & 4,5 & 0,09 & 0,30 & 0,06 & 340 \\
\hline FC80A20 & 5,9 & 0,8 & 13,5 & 0,16 & 0,39 & 0,12 & 270 \\
\hline T80A20 & 6,6 & 1,1 & 18,0 & 0,20 & 0,37 & 0,08 & 350 \\
\hline CP80A20 & 4,4 & 0,4 & 24,0 & 0,39 & 0,30 & 0,07 & 510 \\
\hline
\end{tabular}

T40CP40A20: sustrato control (40\% turba, $40 \%$ corteza de pino, $20 \%$ arena).

SM80A20: tratamiento (80\% musgo Sphagnum, $20 \%$ arena).

FC80A20: tratamiento (80\% fibra de coco, $20 \%$ arena).

T80A20: tratamiento (80\% turba, $20 \%$ arena).

CP80A20: tratamiento (80\% corteza de pino compostada, $20 \%$ arena). 
ácido hay una intensa alteración de minerales y la estructura se vuelve inestable, es por esto que los sustratos más bajos en pH, como CP80A20 afectan negativamente el diámetro, peso seco radical, aéreo $\mathrm{y}$ total. A un $\mathrm{pH}$ inferior a 5 pueden presentarse deficiencias de nitrógeno $(\mathrm{N})$, potasio $(\mathrm{K})$, calcio $(\mathrm{Ca})$, magnesio $(\mathrm{Mg})$ y con valores superiores a 6,5 se disminuye la asimilabilidad de hierro ( $\mathrm{Fe})$, fósforo $(\mathrm{P})$, manganeso $(\mathrm{Mn})$, boro $(\mathrm{B})$, zinc $(\mathrm{Zn})$ y cobre $(\mathrm{Cu})$ (Bauxali y Aguilar, 2002).

El pH óptimo para el cultivo de kiwi corresponde a un valor entre 6,0 y 7,0, por lo cual las mezclas más cercanas a este valor corresponden a FC80A20 y T80A20. El sustrato FC80A20 cumple con los requisitos de $\mathrm{pH}$ óptimos, tanto para Baudoin et al. (2002), como para Zuccherelli y Zuccherelli (1990); sin embargo, esto no fue así para las plántulas de kiwi en las condiciones de este ensayo. Los mejores resultados se obtuvieron en SM80A20, donde se produce un efecto acidificante en la mezcla por efecto del musgo. Respecto a la densidad aparente todos los sustratos presentan una densidad cercana a $400 \mathrm{~kg} \mathrm{~m}^{-3}$, siendo el más liviano el que contiene fibra de coco en un $80 \%$ combinado con $20 \%$ de arena, muy cercano al que contiene musgo o turba en $80 \%$ y un $20 \%$ de arena. La incorporación de corteza de pino aumenta la densidad aparente desde 460 a $510 \mathrm{~kg} \mathrm{~m}^{-3}$ cuando se agrega en 40 a $80 \%$. Los resultados de densidad aparente evaluados están sobre el valor óptimo de $220 \mathrm{~kg} \mathrm{~m}^{-3}$ indicado por Baudoin et al. (2002) y Ansorena (1994). El sustrato FC80A20 muestra el mayor porcentaje de porosidad, el cual alcanzó un $80 \%$. Abad et al. (2005) indican que la fibra de coco presenta ventajas de estabilidad física, porosidad total elevada $(64,1$ a $98,3 \%)$, baja densidad aparente, $(0,03$ a $0,9 \mathrm{~g}$ $\mathrm{cm}^{-3}$ ) y una adecuada aireación $(24,2$ a $89,4 \%)$, en coincidencia con estos resultados.

En relación a la conductividad eléctrica (CE) alcanzada al finalizar el ensayo (Tabla 4), las mezclas FC80A20 y T80A20 alcanzan los niveles óptimos de Baudoin et al. (2002). Sin embargo, FC80A20 no fue apropiado para las plántulas de kiwi, que crecieron menos (Tabla 2). Según Baudoin et al. (2002) la salinidad de un sustrato ideal no debe superar los $200 \mathrm{ppm}$ de sales solubles, cuyo valor de CE equivale a un máximo de $2 \mathrm{mS} \mathrm{cm}^{-1}$, todo lo que sobrepase este nivel a $25^{\circ} \mathrm{C}$, corre el riesgo de ser fitotóxico, lo que dependerá de la capacidad de retención de sales del cultivo. Los sustratos SM80A20 y CP80A20 alcanzan niveles
Tabla 4. Análisis de $\mathrm{pH}$ y conductividad eléctrica en sustratos al finalizar el ensayo.

\begin{tabular}{lcc}
\hline Sustratos & $\mathrm{pH}$ & $\left.\mathrm{CE}(\mathrm{dS} \mathrm{m})^{-1}\right)$ \\
\hline T40CP40A20 (Control) & 6,6 & 0,5 \\
S80A20 & 7,9 & 0,7 \\
FC80A20 & 7,1 & 0,6 \\
T80A20 & 7,4 & 0,7 \\
CP80A20 & 5,9 & 0,3 \\
\hline
\end{tabular}

T40CP40A20: sustrato control (40\% turba, $40 \%$ corteza de pino, $20 \%$ arena).

SM80A20: tratamiento (80\% musgo Sphagnum, $20 \%$ arena). FC80A20: tratamiento (80\% fibra de coco, $20 \%$ arena). T80A20: tratamiento ( $80 \%$ turba, $20 \%$ arena).

ideales según este autor. De acuerdo a valores de conductividad eléctrica adecuados para kiwi, los sustratos T40CP40A20, SM80A20, FC80A20 y CP80A20 son óptimos para este cultivo.

Los valores de materia orgánica (MO), evidencian que ninguno de los sustratos alcanza el valor propuesto por Baudoin et al. (2002), que es mayor a $80 \%$. El contenido de MO para kiwi debe encontrarse entre 3 y $4 \%$, por lo tampoco ninguna de las mezclas ensayadas (Tabla 3 ) se acerca a este nivel. Solo SM80A20 se encontraría cercano a lo requerido por kiwi.

La evaluación de contenidos de nutrientes $\mathrm{N}$, $\mathrm{P}_{2} \mathrm{O}_{5}$ y $\mathrm{K}_{2} \mathrm{O}$ expuestos en la Tabla 3, indican que el mayor contenido de $\mathrm{N}$ se obtuvo en el sustrato CP80A20, donde la corteza de pino aporta el nitrógeno. Sin embargo, de acuerdo a diámetro de tallo, los pesos secos radical, aéreo y total los resultados fueron deficientes, por lo que no es una buena alternativa de remplazo para la turba. Los mayores contenidos de $\mathrm{P}_{2} \mathrm{O}_{5}$ y $\mathrm{K}_{2} \mathrm{O}$ se obtuvieron en el sustrato compuesto por un $80 \%$ de fibra de coco y $20 \%$ de arena. Tal como para la corteza de pino, tampoco en relación a los parámetros de crecimiento, la fibra de coco combinada con arena es una alternativa de remplazo para la turba.

El pH influye en la asimilabilidad de los nutrientes por la planta. Con un $\mathrm{pH}$ inferior a 5 pueden presentarse deficiencias de $\mathrm{N}, \mathrm{K}, \mathrm{Ca}, \mathrm{Mg}$ y con valores superiores a 6,5 se disminuye la asimilabilidad de $\mathrm{Fe}, \mathrm{P}, \mathrm{Mn}, \mathrm{B}, \mathrm{Zn}$ y $\mathrm{Cu}$ (Bauxali y Aguilar, 2002). El sustrato FC80A20 presentaría niveles óptimos de $\mathrm{P}_{2} \mathrm{O}_{5}$ y $\mathrm{K}_{2} \mathrm{O}$. Respecto a valores de nitrógeno total adecuados para el cultivo de kiwi, todos los sustratos ensayados muestran valores inferiores al nivel óptimo, que es de 1,5 a 1,8\%, lo 
que se estandarizó mediante la incorporación del fertilizante de entrega controlada.

Los resultados de los análisis químicos de los sustratos luego de finalizar el ensayo se muestran en la Tabla 4, donde en todos los sustratos ensayados se elevaron el nivel de $\mathrm{pH}$ y conductividad eléctrica respecto al valor inicial respectivo.

Los altos niveles de CE de los sustratos analizados podrían deberse a un alto aporte de sales minerales del agua utilizada en el riego, considerando que los sustratos no contienen alto aporte de sales. Los materiales orgánicos poseen una alta CIC. La capacidad de los sustratos orgánicos para adsorber cationes metálicos depende del $\mathrm{pH}$; cuanto más alto es el pH, más elevada es la CIC (Abad, 1995). Los materiales orgánicos presentan mayor capacidad tampón que los inorgánicos y, por lo tanto, mayor capacidad para mantener constante el pH (Bauxali y Aguilar, 2002). El sustrato T80A20 poseería una mayor capacidad tampón, ya que es donde menos varió el $\mathrm{pH}$, lo que no es válido para las mezclas que incluyen al musgo Sphagnum en remplazo de la turba.

\section{Conclusiones}

Los sustratos compuestos por Sphagnum (80\% $\mathrm{v} / \mathrm{v})$ y arena $(20 \% \mathrm{v} / \mathrm{v})$; fibra de coco $(80 \% \mathrm{v} / \mathrm{v})$ y arena $(20 \% \mathrm{v} / \mathrm{v})$; en comparación al sustrato control compuesto por turba $(40 \% \mathrm{v} / \mathrm{v})$ con corteza de pino $(40 \% \mathrm{v} / \mathrm{v})$ y arena $(20 \% \mathrm{v} / \mathrm{v})$, son adecuados para la producción de almácigos de kiwi y constituyen una alternativa de reemplazo al uso de la turba.

El sustrato control usado en el vivero muestra un $20 \%$ de materia orgánica, una densidad aparente

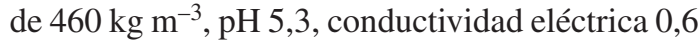
$\mathrm{dS} \mathrm{m}{ }^{-1}, \mathrm{~N} 0,29 \%, \mathrm{P}_{2} \mathrm{O}_{5} 0,25 \%, \mathrm{~K}_{2} \mathrm{O} 0,06 \%$, de estos parámetros solo algunos muestran rangos óptimos para kiwi, por lo que el crecimiento logrado fue medio en comparación con las mezclas constituidas por $80 \%$ v/v de Sphagnum, fibra de coco, corteza de pino o turba, cada una combinada con $20 \%$ de arena.

\section{Literatura Citada}

Abad, M.

1993. Sustratos. Características y propiedades. En: Cultivos sin suelo. Canovas, F., Díaz, J. Ediciones Instituto de Estudios Almerienses. Fundación para la Investigación Agraria en la Provincia de Almería. Almería-España. pp. 47-62.

Abad, M.

1995. Sustratos para el cultivo sin suelo. En: El cultivo del tomate. Nuez, F. Mundi-Prensa. Madrid-España. pp. 131-166.

Abad, M.; Noguera, P.; Puchades, R.; Maquieiria, A; Noguera, N. 2002. Physico-chemical and chemical properties of some coconut coir dists for use as a peat substitute for containerised ornamental plants. Biosource Technology, 82: 241-245.

Abad, M.; Fornes, F.; Garrión, C.; Noguera, V.

2005. Physical properties of various coconut coir dusts compared to peat. HortScience, 40 (7): 2138-2144.

Ansorena, M.

1994. Sustratos. Propiedades y caracterización. Mundi-Prensa. Madrid-España pp. 170 p.

Arrieta, V.; Terés, V.

1993. Caracterización física y química y manejo agronómico de la corteza de pino (Pinus radiata) como sustrato de cultivo. Congreso Forestal Español. Lourizán. Ponencias y comunicaciones Tomo II. Disponible en: http:// secforestales.org/publicaciones/index.php/congresos/ article/viewFile/3248/3213. Consultado 25/Sep/2015.

Baixauli, C.; Aguilar, J.M.

2002. Cultivo sin suelo de hortalizas: aspectos prácticos y experiencias. Serie Divulgativa Técnica. Generalitat Valenciana. Consellería de Agricultura, Pesca y Alimentación. Valencia, España. 110 p.
Baudoin, W.; Grafiadellis, M.; Jiménez, R.; La Malfa, G.; Marínez-García, P.; Nisen, A.; Verlodt, H.; de Vilele, O. von Zabelitz, C.; Garnaud, J.

2002. El cultivo protegido en clima mediterráneo. Estudio FAO Producción y Protección Vegetal 90. Organización de las Naciones Unidas para la Agricultura y Alimentación Roma-Italia. 318 p.

Bures, $\mathrm{S}$.

2002. Sustratos: propiedades físicas, químicas y biológicas. Informes sobre la Industria Hortícola. Revista Horticultura, Extra: 70-78.

Compo Agro.

Ficha técnica de Basacote Plus. http://www.compoexpert.com/fileadmin/user_upload/compo_expert/ cl/documents/pdf/2009_Basacote.pdf Consultado: $11 / 12 / 14$.

Cruz-Crespo, E.; Can-Chulim, A.; Sandoval-Villa, M.; Bugarín-

Montoya, R.; Robles-Bermúdez, A.; Juárez-López P.

2013. Sustratos en la horticultura. Bio Ciencias, 2 (2): 17-26. Díaz, F.

2004. Selección de sustratos para la producción de hortalizas en invernadero. En: Memorias del IV Simposio Nacional de Horticultura. Invernaderos: Diseño, Manejo y Producción. Sánchez, R.A.; Moreno, A.; Puente, Araiza, J. (ed.). Torreón. Coah-México. pp. 42-67.

Gaudig, G.; Fengler, F.; Krebs, M.; Prager, A.; Schulz, J.; Wichmann, S. y Joosten, H.

2014. Sphagnum farming in Germany - a review of progress. Mires and Peat, 13: 1-11.

Hartmann, T.; Kester, D.; Davies, F. and Geneve, R.

2002. Plant propagation. Principles and practices. $6^{\text {th }}$ Edition. Prentice Hall. New Jersey-EE.UU. 770 p. 
Honorato, R.; Bonomelli, C.

1999. Medios artificiales en producción de plantas. El Campesino, 130 (3): 40-43.

Jeangille, $\mathrm{P}$.

1998. Sustratos para la horticultura en regiones tropicales y subtropicales. FAO. Oficina Regional de las Naciones Unidas. Santiago-Chile. 76 p.

Li, H.; Parent, L.; Karam, A.; Tremblay, C.

2004. Potential of Sphagnum peat for improving soil organic matter, water holding capacity, bulk density and potato yield in a sandy soil. Plant and Soil, 265: 355-365.

Olivo, V.; Buduba, C.

2006. Influencia de seis sustratos en el crecimiento de Pinus ponderosa producido en contenedores bajo condiciones de invernáculo. Bosque, 27 (3): 267-271.

Pastor, N.

2003. Influencia del sustrato y del tamaño del contenedor en el trasplante al terreno definitivo de plantas ornamentales cultivadas en contenedor. Actas de Horticultura $\mathrm{N}^{\mathbf{o}} 39$. $X$ Congreso Nacional de Ciencias Hortícolas. Pontevedra, Barcelona-España. 523 p.

Puustjärvi, V.

1994. La turba y su manejo en la horticultura. Reus. Ed. Horticultura. Tarragona-España. 126 p.
Roselló, A; Domínguez, A; Girona, R; Ruiz, M.

1999. Comparación de diversos sustratos para su utilización en viveros ecológicos. Lagascalia, 25: 176-177.

Schmilewski, G.

2008. The role of peat in assuring the quality of growing media. Mires and Peats, 3: 1-9.

Schofield, W.B.

1985. The peat mosses-subclass Sphagnidae. En: Introduction to Bryology. Schofield, W. The Blackburn Press. Caldwell, New Jersey-EE.UU. pp. 32-48.

Schrader, W.

2003. Using transplants in vegetable production. University of California. Division of Agricultural and Natural Resources. Publication 8013. Disponible en: http://anrcatalog.ucanr. edu/pdf/8013.pdf Consultado: 22/Feb/2016.

Sudzuki, F.

2002. Cultivo de frutales menores. $7^{\text {a }}$ edición. Editorial Universitaria. Santiago-Chile. 184 p.

Zapata, N.; Guerrero, F.; Polo, A.

2005. Evaluación de corteza de pino y residuos urbanos como componentes de sustratos de cultivo. Agricultura Técnica, 65 (4): 387-387.

Zuccherelli, G.; Zuccherelli G.

1990. La Actinidia (kiwi). $2^{\text {a }}$ edición. Editorial Mundi-Prensa. Madrid España. 228 p. 
